\title{
A search for molecules in damped Lyman-alpha absorbers occulting millimetre-loud quasars ${ }^{\star}$
}

\author{
S. J. Curran ${ }^{1}$, M. T. Murphy ${ }^{1}$, J. K. Webb ${ }^{1}$, F. Rantakyrö ${ }^{2,3}$, L. E. B. Johansson ${ }^{4}$, and S. Nikolić, ${ }^{4,5}$ \\ 1 School of Physics, University of New South Wales, Sydney NSW 2052, Australia \\ 2 European Southern Observatory, Casilla 19001, Santiago 19, Chile \\ 3 Observatorio Cerro Calan, Universidad de Chile, Santiago, Chile \\ ${ }^{4}$ Onsala Space Observatory, Chalmers University of Technology, 43992 Onsala, Sweden \\ 5 Astronomical Observatory, Volgina 7, 11160 Belgrade, Serbia
}

Received 21 June 2002 / Accepted 27 August 2002

\begin{abstract}
We have used the SEST 15-metre and Onsala 20-metre telescopes to perform deep (rms $\gtrsim 30 \mathrm{mJy}$ ) integrations of various molecular rotational transitions towards damped Lyman-alpha absorption systems (DLAs) known to occult millimetreloud quasars. We have observed 6 new systems and improved the existing limits for 11 transitions. These limits may be approaching the sensitivities required to detect new systems and we present a small number of candidate systems which we believe warrant further observation.
\end{abstract}

Key words. quasars: absorption lines - cosmology: observations - cosmology: early Universe

\section{Introduction}

Millimetre-band molecular absorption systems along the lineof-sight toward quasars can provide a powerful probe of cold gas in the early Universe. Wiklind \& Combes (1994, 1995,1996 b) have used such absorption lines to study a variety of properties of the absorbers themselves (e.g. relative column densities, kinetic and excitation temperatures, filling factors). Constraints on the cosmic microwave background temperature can also be obtained by comparing the optical depths of different rotational transitions (e.g. Wiklind \& Combes 1996a). If the background quasar is gravitationally lensed, time delay studies can yield constraints on the Hubble constant (e.g. Wiklind \& Combes 2001). However, these studies have so far been limited by the paucity of mmband molecular absorbers: only 4 such systems are currently known-towards TXS 0218+357 (Wiklind \& Combes 1995), PKS 1413+135 (Wiklind \& Combes 1997), TXS 1504+377 (Wiklind \& Combes 1996c) and PKS 1830-211 (Wiklind \& Combes 1998).

Recent attention has focused on using molecular absorption lines as a probe for possible changes in the fundamental constants on cosmological time-scales. Detailed studies of the relative positions of heavy element optical transitions compared with laboratory spectra favour a smaller fine structure constant $\left(\alpha \equiv e^{2} / \hbar c\right)$ at redshifts $0.5<z<3.5$ at the $4.1 \sigma$

Send offprint requests to: $\mathrm{S}$. Curran,

e-mail: sjc@bat.phys.unsw.edu.au

* Based on results collected at Onsala Space Observatory, Sweden and the European Southern Observatory, La Silla, Chile. significance level (Murphy et al. 2001b; Webb et al. 2001). The observed fractional change in $\alpha\left[\Delta \alpha / \alpha=(-0.72 \pm 0.18) \times 10^{-5}\right]$ is very small, so systematic errors have to be carefully considered. However, a thorough search for systematics has not revealed a simpler explanation of the optical results (Murphy et al. 2001c) and so independent constraints at similar redshifts are required.

Comparison of molecular rotational (i.e. mm-band) and corresponding $\mathrm{H}_{\mathrm{I}} 21-\mathrm{cm}$ absorption line frequencies has the potential to constrain changes in $\alpha$ with a fractional precision $\sim 10^{-6}$ per absorption system. The ratio of the hyperfine $\left(\mathrm{HI}_{\mathrm{I}}\right)$ transition frequency to that of a molecular rotational line is $\propto y \equiv \alpha^{2} g_{\mathrm{p}}$ and so any variation in this will be observed as a difference in the apparent redshifts of these lines (Drinkwater et al. 1998).

From spectra of two of the known high redshift absorbers, PKS 1413+135 and TXS 0218+357, Carilli et al. (2000) and Murphy et al. (2001a) have obtained constraints on $\Delta y / y$ consistent with zero $y$-variation at redshifts $z_{\text {abs }}=0.6847$ and 0.24671 , respectively. However, the major uncertainty in the $\mathrm{mm} / \mathrm{H}$ I comparison is that intrinsic velocity differences between the $\mathrm{mm}$ and $\mathrm{HI}_{\mathrm{I}}$ absorption lines can be introduced if the lines-of-sight to the millimetre wave and radio continuum emission regions of the quasar differ, as is certainly the case for PKS $1413+135$ and TXS $0218+357$ (Carilli et al. 2000). Thus, a statistical sample of $\mathrm{mm} / \mathrm{H}$ I comparisons is required to independently check the optical results.

One systematic approach to finding new high redshift molecular absorbers is to target high column density systems with known redshifts. A convenient sample is therefore the 
Table 1. Top: the northern sample. The radio flux densities at various $\mathrm{GHz}$ frequencies of the background quasars are given (see Curran et al. 2002 for details). Note that a " $\approx$ " denotes a variable flux density. $z_{\mathrm{abs}}$ is the DLA redshift and the final column gives the molecules most commonly detected in the four known absorbers (see Wiklind \& Combes references), which fall into the Onsala $84-116 \mathrm{GHz}^{\mathrm{band}}$ ( ${ }^{*} \mathrm{HCO}{ }^{+}$ $0 \rightarrow 1$ in B $08279+5255$ and ${ }^{*} \mathrm{CS} 2 \rightarrow 3$ in B 1017+1055 are $7 \mathrm{~mm}$ observations). Note that CS has so far only been detected in PKS $1830-211$ (Wiklind \& Combes 1996c) but since none of the commonly detected molecules fell into the band at the DLA redshift we tuned to this dense gas tracer. Bottom: the southern sample. The final column lists the molecules most commonly detected which fall into the SEST 78-116 GHz and $128-170 \mathrm{GHz}$ bands.

\begin{tabular}{|c|c|c|c|c|c|c|c|c|c|c|}
\hline \multirow[t]{2}{*}{ Quasar } & \multicolumn{2}{|c|}{ Coordinates (J2000) } & \multicolumn{6}{|c|}{ Radio flux densities (Jy) } & \multirow[t]{2}{*}{$z_{\mathrm{abs}}$} & \multirow[t]{2}{*}{ Transition } \\
\hline & $\mathrm{h} \mathrm{m} \mathrm{s}$ & $\mathrm{d}^{\prime}{ }^{\prime \prime}$ & $S_{1.4}$ & $S_{5.0}$ & $S_{22}$ & $S_{90}$ & $S_{230}$ & $S_{350}$ & & \\
\hline B $0235+1624$ & 023838.9 & 163659 & 2.36 & 1.64 & 2.48 & $\approx 1-6$ & $\approx 1-4$ & - & 0.523869 & $\mathrm{CS} 2 \rightarrow 3$ \\
\hline В $0248+430$ & 025134.5 & 431516 & 1.43 & 0.66 & $\approx 0.7$ & 0.32 & 0.08 & - & 0.3939 & $\mathrm{CS} 2 \rightarrow 3$ \\
\hline B $0738+313$ & 074110.7 & 311200 & 2.05 & - & $\approx 1.3$ & 0.27 & 0.10 & - & 0.2212 & $\mathrm{CO} 0 \rightarrow 1$ \\
\hline B $0827+243$ & 083052.1 & 241100 & 0.84 & 0.89 & $\approx 1.3$ & 2.4 & $\approx 1.3$ & - & 0.5247 & $\mathrm{CS} 2 \rightarrow 3$ \\
\hline В $08279+5255$ & 083141.6 & 524518 & 0.001 & - & - & - & - & 0.08 & 2.97364 & ${ }^{*} \mathrm{HCO}^{+} 0 \rightarrow 1$ \\
\hline$\ldots$ & $\ldots$ & $\ldots$ & $\ldots$ & $\ldots$ & $\ldots$ & $\ldots$ & $\ldots$ & $\ldots$ & $\ldots$ & $\mathrm{CO} 2 \rightarrow 3$ \\
\hline B $1017+1055$ & 102008.8 & 104003 & 0.22 & - & - & - & 0.004 & - & 2.380 & ${ }^{*} \mathrm{CS} 2 \rightarrow 3$ \\
\hline$\ldots$ & $\ldots$ & $\ldots$ & $\ldots$ & $\ldots$ & $\ldots$ & $\ldots$ & $\ldots$ & $\ldots$ & $\ldots$ & $\mathrm{CO} 2 \rightarrow 3$ \\
\hline B $1328+307$ & 133108.3 & 303033 & 14.7 & 1.41 & 2.7 & 0.75 & 0.33 & - & 0.69218 & $\mathrm{HCO}^{+} 1 \rightarrow 2$ \\
\hline$\cdots$ & $\cdots$ & $\cdots$ & $\ldots$ & $\cdots$ & $\cdots$ & $\ldots$ & $\ldots$ & $\ldots$ & $\ldots$ & $\mathrm{CS} 2 \rightarrow 3$ \\
\hline В $0458-020$ & 050112.8 & -015914 & 2.2 & $\approx 3$ & - & 0.8 & $\approx 0.5$ & - & 2.0399 & $\mathrm{HCO}^{+} 2 \rightarrow 3$ \\
\hline$\ldots$ & $\ldots$ & $\ldots$ & $\ldots$ & $\ldots$ & $\ldots$ & $\ldots$ & $\ldots$ & $\ldots$ & $\ldots$ & $\mathrm{CO} 3 \rightarrow 4$ \\
\hline В $0834-201$ & 083639.2 & -201659 & 1.97 & 1.5 & - & 0.85 & 0.28 & - & 1.715 & $\mathrm{HCO}^{+} 2 \rightarrow 3$ \\
\hline$\ldots$ & $\ldots$ & $\ldots$ & $\ldots$ & $\ldots$ & $\ldots$ & $\ldots$ & $\ldots$ & $\ldots$ & $\ldots$ & $\mathrm{CO} 3 \rightarrow 4$ \\
\hline В 1229-0207 & 123200.0 & -022405 & 1.90 & 0.90 & $\approx 1$ & $\approx 0.4$ & $\approx 0.2$ & - & 0.3950 & $\mathrm{CO} 0 \rightarrow 1$ \\
\hline$\ldots$ & $\ldots$ & $\ldots$ & $\ldots$ & $\ldots$ & $\ldots$ & $\ldots$ & $\ldots$ & $\ldots$ & $\ldots$ & $\mathrm{CO} 1 \rightarrow 2$ \\
\hline B $1451-375$ & 145427.4 & -374733 & 1.57 & 1.84 & - & $\approx 1.5$ & $\approx 0.5$ & - & 0.2761 & $\mathrm{CO} 0 \rightarrow 1$ \\
\hline$\ldots$ & $\ldots$ & $\ldots$ & $\ldots$ & $\ldots$ & $\ldots$ & $\ldots$ & $\ldots$ & $\ldots$ & $\ldots$ & $\mathrm{HCO}^{+} 1 \rightarrow 2$ \\
\hline
\end{tabular}

damped Lyman-alpha absorbers defined to have neutral hydrogen column densities $N_{\mathrm{HI}} \gtrsim 10^{20} \mathrm{~cm}^{-2}$. We therefore compiled a catalogue of all known DLAs and shortlisted those which are illuminated by radio-loud quasars (Curran et al. 2002) ${ }^{1}$. Of these, seven in the northern sky have measured millimetre fluxes, and in the south there are around a dozen DLAs illuminated by millimetre-loud quasars, of which we observed the four loudest (Table 1).

We note that Wiklind \& Combes (1994b, 1995, 1996b) searched with null results for redshifted molecular emission and absorption towards 12 and 46 quasars, respectively. However, only 11 are occulted by DLAs and not all of these are radio-loud (Table 2). This motivates a more systematic search for millimetre absorption systems associated with DLAs. In this paper we present the results of our first search: the DLAs which occult known millimetre-loud quasars with the SEST ${ }^{2}$ and Onsala 20-m telescopes.

\section{Observations}

The northern $3 \mathrm{~mm}$ and $7 \mathrm{~mm}$ observations were performed in February and April 2002, respectively, with the 20-m telescope at Onsala Space Observatory, Sweden. For the $3 \mathrm{~mm}$ observations the SIS receiver was tuned to single-sideband mode and the backend was a hybrid correlator with a bandwidth of

\footnotetext{
1 Available from http://www.phys. unsw.edu.au/ sjc/dla

2 The Swedish-ESO Sub-millimetre Telescope is operated jointly by ESO and the Swedish National Facility for Radio Astronomy, Onsala Space Observatory, Chalmers University of Technology.
}

$1280 \mathrm{MHz}$ and a channel separation of $0.8 \mathrm{MHz}$. We used dualbeam switching with a throw of $12^{\prime}$ in azimuth, and pointing errors were typically $3^{\prime \prime}$ rms on each axis. The intensity was calibrated using the chopper-wheel method and typical system temperatures, on the $T_{\mathrm{A}}^{*}$-scale, were around $300 \mathrm{~K}$. For the $7 \mathrm{~mm}$ observations we used the $43 \mathrm{GHz}$ SIS receiver and again the backend was the hybrid correlator with a bandwidth of $640 \mathrm{MHz}$ and a channel separation of $0.4 \mathrm{MHz}$. Since there is no beam switch capability at these frequencies, we removed the ripple caused by standing waves by subtracting the Fourier components in a transform of the spectra. Typical system temperatures, on the $T_{\mathrm{A}}^{*}$-scale, were around $200 \mathrm{~K}$.

The southern sources in the sample were observed in April 2002 with the 15-m SEST at La Silla, Chile, using the $100 \mathrm{GHz}$ and $150 \mathrm{GHz}$ SESIS receivers. These were tuned to singlesideband mode and typical system temperatures, on the $T_{\mathrm{A}}^{*}$ scale, were $\approx 200 \mathrm{~K}$ for the $\mathrm{RX} 100$ and $\approx 250 \mathrm{~K}$ for the RX150. The backends were acousto-optic spectrometers with 1440 channels and a channel width of $0.7 \mathrm{MHz}$. As with the Onsala $3 \mathrm{~mm}$ observations, we used dual-beam switching with a throw of about $12^{\prime}$ in azimuth, and pointing errors were typically $2^{\prime \prime}$ rms on each axis. Again, the intensity was calibrated using the chopper-wheel method.

\section{Results and discussion}

Upon the removal of a low order baseline and subsequent averaging of the data for each quasar, no absorption features 
Table 2. Molecular lines previously searched for in radio-illuminated DLAs. All of the observations were performed with the IRAM 30-m telescope, except for $\mathrm{HCO}^{+} 2 \rightarrow 3$ towards B 0458-020 which was observed with the SEST. Again, apart from those marked “*” which are from Wiklind \& Combes (1995), the radio flux densities are from the various sources cited in Curran et al. (2002). $\sigma$ is the lowest rms noise obtained for this frequency (with the reference given) recalculated for a channel width of $10 \mathrm{~km} \mathrm{~s}^{-1}$ (see footnote 3). Note that Wiklind \& Combes (1994b) is a search for CO emission, although any absorption features at the appropriate redshift should be apparent.

\begin{tabular}{|c|c|c|c|c|c|c|c|c|c|}
\hline \multirow[t]{2}{*}{ Quasar } & \multirow[t]{2}{*}{$z_{\text {abs }}$} & \multirow[t]{2}{*}{ Transition } & \multicolumn{5}{|c|}{ Radio flux densities (Jy) } & \multirow[t]{2}{*}{$\sigma[\mathrm{mK}]$} & \multirow[t]{2}{*}{ Reference } \\
\hline & & & $S_{1.4}$ & $S_{5.0}$ & $S_{22}$ & $S_{90}$ & $S_{230}$ & & \\
\hline \multirow[t]{2}{*}{ B $0235+1624$} & 0.5238 & $\mathrm{CO} 1 \rightarrow 2$ & 2.36 & 1.64 & 2.48 & $1.2^{*}$ & $1.3^{*}$ & 2.3 & Wiklind \& Combes (1995) \\
\hline & & $\mathrm{HCO}^{+} 3 \rightarrow 4$ & $\ldots$ & $\ldots$ & $\ldots$ & $\ldots$ & $\ldots$ & 3.6 & Wiklind \& Combes (1995) \\
\hline \multirow[t]{2}{*}{ В $0458-020$} & 2.0397 & $\mathrm{CO} 2 \rightarrow 3$ & 2.2 & $\approx 3$ & - & 0.8 & $\approx 0.5$ & 3.0 & Wiklind \& Combes (1994b) \\
\hline & & $\mathrm{HCO}^{+} 2 \rightarrow 3$ & $\ldots$ & $\ldots$ & $\ldots$ & $\ldots$ & $\ldots$ & 7.1 & Wiklind \& Combes (1996b) \\
\hline B $0528-2505$ & 2.1408 & $\mathrm{CO} 2 \rightarrow 3$ & 1.50 & 1.13 & - & - & - & 1.3 & Wiklind \& Combes (1994b) \\
\hline \multirow[t]{2}{*}{ В 0834-201 } & 1.715 & $\mathrm{HCO}^{+} 2 \rightarrow 3$ & - & 1.5 & - & 0.85 & 0.28 & 3.6 & Wiklind \& Combes (1996b) \\
\hline & & $\mathrm{HCO}^{+} 3 \rightarrow 4$ & $\ldots$ & $\ldots$ & $\ldots$ & $\ldots$ & $\ldots$ & 3.9 & Wiklind \& Combes (1996b) \\
\hline B $1215+333$ & 1.9984 & $\mathrm{CO} 2 \rightarrow 3$ & 0.18 & 0.08 & - & - & - & 4.4 & Wiklind \& Combes (1994b) \\
\hline B $1229-0207$ & 0.39498 & $\mathrm{CO} 1 \rightarrow 2$ & 1.90 & 0.90 & $\approx 1$ & $\approx 0.4$ & $\approx 0.2$ & 12 & Wiklind \& Combes (1995) \\
\hline \multirow[t]{3}{*}{ B $1328+307$} & 0.69215 & $\mathrm{CO} 1 \rightarrow 2$ & 14.7 & 1.41 & 2.7 & $0.6^{*}$ & $0.24^{*}$ & 6.1 & Wiklind \& Combes (1995) \\
\hline & & $\mathrm{CO} 2 \rightarrow 3$ & $\ldots$ & $\ldots$ & $\ldots$ & $\ldots$ & $\ldots$ & 6.5 & Wiklind \& Combes (1995) \\
\hline & & $\mathrm{HCO}^{+} 1 \rightarrow 2$ & $\ldots$ & $\ldots$ & $\ldots$ & $\ldots$ & $\ldots$ & 5.8 & Wiklind \& Combes (1995) \\
\hline \multirow[t]{4}{*}{ B $2136+142$} & 2.1346 & $\mathrm{CO} 2 \rightarrow 3$ & - & 1.11 & 1.6 & $0.59^{*}$ & 0.25 & 2.8 & Wiklind \& Combes (1996b) \\
\hline & & $\mathrm{CO} 3 \rightarrow 4$ & $\ldots$ & $\ldots$ & $\ldots$ & $\ldots$ & $\ldots$ & 2.3 & Wiklind \& Combes (1996b) \\
\hline & & $\mathrm{CO} 5 \rightarrow 6$ & $\ldots$ & $\ldots$ & $\ldots$ & $\ldots$ & $\ldots$ & 3.9 & Wiklind \& Combes (1996b) \\
\hline & & $\mathrm{HCO}^{+} 2 \rightarrow 3$ & $\ldots$ & $\ldots$ & $\ldots$ & $\ldots$ & $\ldots$ & 3.4 & Wiklind \& Combes (1995) \\
\hline
\end{tabular}

of $\geq 3 \sigma /$ channel were found $^{3}$. In Table 3 we summarise the derived upper limits together with the previously published results. Comparing the optical depth limits with those in the four known absorbers, the strongest absorber, towards PKS 1830 211 , has $\tau_{\mathrm{mm}} \approx 1$ for $\mathrm{HCO}^{+} 1 \rightarrow 2$ and $\tau_{\mathrm{mm}} \approx 0.5$ for $\mathrm{HCO}^{+}$ $2 \rightarrow 3$. The weakest, towards PKS $1413+135$, has $\tau_{\mathrm{mm}} \approx 0.1$ for both $\mathrm{CO} 0 \rightarrow 1$ and $\mathrm{HCO}^{+} 2 \rightarrow 3$. The two remaining systems have optical depths similar to that towards PKS 1830211 and so our new observations and the previous surveys should have been sensitive to (at least) $\mathrm{CO}$ and $\mathrm{HCO}^{+}$at similar strengths to those in 3 of the 4 known systems. Only for the observations of B $0235+1624, \mathrm{HCO}^{+} 2 \rightarrow 3$ towards B $2136+141$ (IRAM) and CO $0 \rightarrow 1$ towards B 1451-375 are the surveys sensitive enough, particularly for resolutions of $\gtrsim 10 \mathrm{~km} \mathrm{~s}^{-1}$, to detect an absorption system of a strength similar to that towards PKS 1413+135 (see Table 4).

From the optical depths we may estimate column density limits for each transition. In Table 3 we give the $3 \sigma$ optical depth limits according to a velocity resolution of $10 \mathrm{~km} \mathrm{~s}^{-1}$ as well as at a resolution of $1 \mathrm{~km} \mathrm{~s}^{-1}$. We give the former in order to show the limits corresponding to a visual inspection of the spectra $^{3}$ and facilitate a more direct comparison with the previous surveys. By multiplying the latter with the expected width of a line, the velocity integrated optical depth for a $3 \sigma$ detection at $\Delta v=1 \mathrm{~km} \mathrm{~s}^{-1}$ is obtained. For all of the limits, assuming LTE conditions, we can estimate the total column density of each transition from

$N_{\mathrm{mm}}=\frac{8 \pi}{c^{3}} \frac{v^{3} \cdot f}{g_{\mu} A_{\mu}} \int \tau \mathrm{d} v$,

${ }^{3}$ See http://www.phys. unsw.edu.au/ sjc/dla-fig1.ps.gz for the spectra and corresponding rms noise levels. where $v$ is the rest frequency of the $J \rightarrow J+1$ transition, $g_{\mu}$ and $A_{\mu}$ are the statistical weight and the Einstein $A$-coefficient of the transition, $\int \tau \mathrm{d} v$ is the velocity integrated optical depth and $f$ is the product of the partition function for an excitation temperature, $T_{x}$ (assumed $\left.\approx 10 \mathrm{~K}\right)$, and $\mathrm{e}^{E_{\mathrm{J}} / k T_{x}} /\left(1-\mathrm{e}^{-h v / k T_{x}}\right)$ (see Wiklind \& Combes 1995, 1996b, 1999 for details). From the optical depth limits, we derive limits on the total column density per unit velocity, thus normalising the limits which depend on the spectral resolution to which the data have been smoothed.

Since we have only upper limits, and thus no knowledge of the width of any line which may be hidden in the noise, as well as uncertainties in the conversion to $\mathrm{H}_{2}$ column densities for molecules other than $\mathrm{CO}$, it is difficult to draw meaningful comparisons between $N_{\mathrm{HI}}$ and $N_{\mathrm{mm}}$. However, by assuming an absorption line of $F W H M \sim 20 \mathrm{~km} \mathrm{~s}^{-1}$ (as in the case of 3 of the 4 known absorbers), we estimate for the lowest optical depth limit (CO $1 \rightarrow 2$ in B $0235+1624$ ) a value of $N_{\mathrm{CO}} \lesssim 6 \times 10^{15} \mathrm{~cm}^{-2}$ at the $3 \sigma$ level and a resolution of $1 \mathrm{~km} \mathrm{~s}^{-1}$, i.e. $N_{\mathrm{CO}} / N_{\mathrm{HI}} \approx 2 \times 10^{-6}$. According to $N_{\mathrm{H}_{2}} \sim 10^{4} N_{\mathrm{CO}}$ (e.g. Wiklind \& Combes 1998), the molecular to atomic hydrogen column density ratio is $N_{\mathrm{H}_{2}} \lesssim 2 \% N_{\mathrm{HI}}$, which is similar to the lower limit estimated from the optical detection of molecular hydrogen ${ }^{4}$ in the DLA towards B 0528-2505

\footnotetext{
${ }^{4}$ In the case of $z>1.8$ sources, the ultra-violet lines of $\mathrm{H}_{2}$ are redshifted into the optical window, making molecular hydrogen readily observable at these frequencies. As well as towards B 0528-2505 (Foltz et al.1988) molecular hydrogen has also been detected in the DLAs occulting the radio-quiet quasars B 0000-2620 (Levshakov et al. 2000), B 0013-0029 (Ge \& Bechtold 1997; Petitjean et al. 2002), B 0347-3819 (Levshakov et al. 2002), B 1232+0815 (Ge \& Bechtold 1997; Srianand et al. 2000) and the inferred (Wolfe et al. 1995) DLA towards B 0551-3637 (Ledoux et al. 2002).
} 
Table 3. Summary of our (Table 1) and the previously published results (Table 2). $V$ is the visual magnitude of the background quasar, $N_{\mathrm{HI}}\left[\mathrm{cm}^{-2}\right]$ is the DLA column density from the Lyman-alpha line and $\tau_{21 \mathrm{~cm}}$ is the optical depth of the redshifted $21 \mathrm{~cm} \mathrm{H}_{\mathrm{I}}$ line (see Curran et al. 2002). The optical depth of the relevant millimetre line is calculated from $\tau=-\ln \left(1-3 \sigma_{\text {rms }} / S_{\text {cont }}\right)$, where $\sigma_{\text {rms }}$ is the rms noise level at a given resolution and $S_{\text {cont }}$ is the continuum flux density, estimated from the values at the neighbouring frequencies (Tables 1 and 2). This is done for resolutions of $10 \mathrm{~km} \mathrm{~s}^{-1}\left[\tau_{\mathrm{mm}}(10)\right]$ and $1 \mathrm{~km} \mathrm{~s}^{-1}\left(\tau_{\mathrm{mm}}\right)$, where for the latter we quote only the best existing limit. For all optical depths, $3 \sigma$ upper limits are quoted and "-" designates where $3 \sigma>S_{\text {cont }}$, thus not giving a meaningful value for this limit. Blanks in the $\tau_{21} \mathrm{~cm}$ field signify that there are no published $\mathrm{H}_{\mathrm{I}}$ absorption data for these DLAs. The final column gives the best existing limit of the column density per unit velocity estimated for the transition $\left[\mathrm{cm}^{-2}\left(\mathrm{~km} \mathrm{~s}^{-1}\right)^{-1}\right]$ (see main text).

\begin{tabular}{|c|c|c|c|c|c|c|c|c|}
\hline DLA & $z_{\mathrm{abs}}$ & Transition & $V$ & $N_{\mathrm{HI}}$ & $\tau_{21 \mathrm{~cm}}$ & $\tau_{\mathrm{mm}}(10)$ & $\tau_{\mathrm{mm}}$ & $N_{\mathrm{mm}} / \Delta v$ \\
\hline B $0235+1624$ & $0.52398^{*}$ & $\mathrm{CO} 1 \rightarrow 2$ & 15.5 & $4 \times 10^{21}$ & $0.05-0.5^{a}$ & $<0.03$ & $<0.09$ & $<3 \times 10^{14}$ \\
\hline$\ldots$ & $\ldots$ & $\mathrm{HCO}^{+} 3 \rightarrow 4$ & $\ldots$ & $\ldots$ & $\ldots$ & $<0.03$ & $<0.3$ & $<4 \times 10^{12}$ \\
\hline$\ldots$ & 0.523869 & $\mathrm{CS} 2 \rightarrow 3$ & $\ldots$ & $\ldots$ & $\ldots$ & $<0.2$ & $<0.9$ & $<2 \times 10^{13}$ \\
\hline В $0248+430$ & 0.3939 & $\mathrm{CS} 2 \rightarrow 3$ & 17.7 & $4 \times 10^{21}$ & 0.20 & $<3$ & - & - \\
\hline B $0458-020$ & $2.0397^{*} / 9$ & $\mathrm{HCO}^{+} 2 \rightarrow 3$ & 18.4 & $5 \times 10^{21}$ & $0.30^{b}$ & $<0.3 /<0.2$ & $<0.4$ & $<2 \times 10^{12}$ \\
\hline$\ldots$ & $2.0397^{*}$ & $\mathrm{CO} 2 \rightarrow 3$ & $\ldots$ & $\ldots$ & $\ldots$ & $<0.4$ & - & - \\
\hline$\ldots$ & 2.0399 & $\mathrm{CO} 3 \rightarrow 4$ & $\ldots$ & $\ldots$ & $\ldots$ & $<0.1$ & $<1$ & $<2 \times 10^{16}$ \\
\hline В $0528-2505$ & 2.1408 & $\mathrm{CO} 2 \rightarrow 3$ & 19.0 & $4 \times 10^{20}$ & $<0.2$ & \multicolumn{3}{|c|}{ No published millimetre fluxes } \\
\hline B $0738+313$ & 0.2212 & $\mathrm{CO} 0 \rightarrow 1$ & 16.1 & $2 \times 10^{21}$ & 0.07 & $<1$ & - & - \\
\hline B $0827+243$ & 0.5247 & $\mathrm{CS} 2 \rightarrow 3$ & 17.3 & $2 \times 10^{20}$ & 0.007 & $<0.1$ & $<0.4$ & $<7 \times 10^{12}$ \\
\hline B $08279+5255$ & 2.97364 & $\mathrm{HCO}^{+} 0 \rightarrow 1$ & 15.2 & $1 \times 10^{20}$ & \multirow{3}{*}{$\cdots$} & $-^{c}$ & - & - \\
\hline$\ldots$ & $\ldots$ & $\mathrm{CO} 2 \rightarrow 3$ & $\ldots$ & $\ldots$ & & $-^{c}$ & - & - \\
\hline В 0834-201 & 1.715 & $\mathrm{HCO}^{+} 2 \rightarrow 3$ & 18.5 & $3 \times 10^{20}$ & & $<0.1 /<0.2$ & $<0.4$ & $<2 \times 10^{12}$ \\
\hline$\ldots$ & $\ldots$ & $\mathrm{HCO}^{+} 3 \rightarrow 4$ & $\ldots$ & $\ldots$ & $\cdots$ & $<0.2$ & $<0.6$ & $<8 \times 10^{12}$ \\
\hline$\ldots$ & $\ldots$ & $\mathrm{CO} 3 \rightarrow 4$ & $\ldots$ & $\ldots$ & $\ldots$ & - & - & - \\
\hline B $1017+1055$ & 2.380 & $\mathrm{CS} 2 \rightarrow 3$ & 17.2 & $8 \times 10^{19}$ & \multirow{4}{*}{$\cdots$} & $-^{d}$ & - & - \\
\hline$\ldots$ & $\ldots$ & $\mathrm{CO} 2 \rightarrow 3$ & $\ldots$ & .. & & $-^{d}$ & - & - \\
\hline B $1215+333$ & 1.9984 & $\mathrm{CO} 2 \rightarrow 3$ & 18.1 & $1 \times 10^{21}$ & & $-{ }_{-}^{I, e}$ & - & - \\
\hline B 1229-0207 & 0.3950 & $\mathrm{CO} 0 \rightarrow 1$ & 16.8 & $1 \times 10^{21}$ & & $<0.3$ & $<1$ & $<6 \times 10^{15}$ \\
\hline$\ldots$ & $\ldots$ & $\mathrm{CO} 1 \rightarrow 2$ & $\ldots$ & $\ldots$ & $\ldots$ & $<1$ & - & - \\
\hline$\ldots$ & $0.39498^{*}$ & $\mathrm{CO} 1 \rightarrow 2$ & $\ldots$ & $\ldots$ & $\ldots$ & - & - & - \\
\hline B $1328+307$ & 0.69215 & $\mathrm{HCO}^{+} 1 \rightarrow 2$ & 17.3 & $2 \times 10^{21}$ & 0.11 & $<0.2 /<0.4$ & $<0.7$ & $<2 \times 10^{12}$ \\
\hline$\ldots$ & $\ldots$ & $\mathrm{CS} 2 \rightarrow 3$ & $\ldots$ & $\ldots$ & $\ldots$. & $<0.3$ & $<2$ & $<4 \times 10^{13}$ \\
\hline$\ldots$ & $\ldots$ & $\mathrm{CO} 1 \rightarrow 2$ & $\ldots$ & $\ldots$ & $\ldots$ & $<0.3$ & $<1$ & $<3 \times 10^{15}$ \\
\hline$\ldots$ & $\ldots$ & $\mathrm{CO} 2 \rightarrow 3$ & $\ldots$ & $\ldots$ & $\ldots$ & $<0.6$ & - & - \\
\hline B $1451-375$ & 0.2761 & $\mathrm{HCO}^{+} 1 \rightarrow 2$ & 16.7 & $1 \times 10^{20}$ & $<0.006$ & $<0.2$ & $<0.6$ & $<2 \times 10^{12}$ \\
\hline$\ldots$ & $\ldots$ & $\mathrm{CO} 0 \rightarrow 1$ & $\ldots$ & $\ldots$ & \multirow[t]{2}{*}{$\ldots$} & $<0.09$ & $<0.3$ & $<2 \times 10^{15}$ \\
\hline B $2136+141$ & 2.1346 & $\mathrm{HCO}^{+} 2 \rightarrow 3$ & 18.9 & $6 \times 10^{19}$ & & $<0.08$ & $<0.3$ & $<1 \times 10^{12}$ \\
\hline$\ldots$ & $\ldots$ & $\mathrm{CO} 2 \rightarrow 3$ & $\ldots$ & $\ldots$ & $\ldots$ & $<0.2$ & $<0.6$ & $<4 \times 10^{15}$ \\
\hline$\ldots$ & $\ldots$ & $\mathrm{CO} 3 \rightarrow 4$ & $\ldots$ & $\ldots$ & $\ldots$ & $<0.2$ & $<0.8$ & $<2 \times 10^{1 \epsilon}$ \\
\hline$\ldots$ & $\ldots$ & $\mathrm{CO} 5 \rightarrow 6$ & $\ldots$ & $\ldots$ & $\ldots$ & $<0.6$ & - & - \\
\hline
\end{tabular}

Notes: where we have observed towards the same quasar ${ }^{*}$ denotes Wiklind \& Combes results (see Table 2 for details). ${ }^{a} \mathrm{H}$ i absorption at $z=0.52385$ (Briggs \& Wolfe 1983). ${ }^{b} \mathrm{H}_{\mathrm{I}}$ absorption at $z=2.03945$ (Wolfe et al. 1985; Briggs et al. 1989). ${ }^{c}$ Assumed flat spectrum, i.e. $S_{45}=S_{87}=S_{350}=0.08 \mathrm{Jy} .{ }^{d}$ Assumed $S_{44} \approx 0.015 \mathrm{Jy}$ and $S_{102} \approx 0.008 \mathrm{Jy} ;{ }^{e} S_{115} \approx 0.011 \mathrm{Jy}$, based on the two available measured values (Table 1).

(Carilli et al. 1996). That is, although we have no clear detections of any molecular absorption lines, the current limits may be approaching those sufficiently low in order to detect molecular absorption in damped Lyman-alpha systems.

Bearing this in mind, we then analysed the spectra for tentative features within a range corresponding to the uncertainty in the DLA redshift ${ }^{5}$. In each case the continuum level was

\footnotetext{
5 Although we have searched for possible absorption lines close to the expected redshifts, it may possible that the line-of-sight to the $\mathrm{mm}$ band and optical emission regions of the quasars may differ $\left(\delta_{\mathrm{LOS}}>0\right)$, giving rise to intrinsic offsets between any millimetre absorption and the known DLA redshifts. Drinkwater et al. (1998) compared millimetre and $\mathrm{H}_{\mathrm{I}} 21-\mathrm{cm}$ Galactic absorption profiles and found that the
}

defined using a polynomial baseline fit and for each channel we generated a $1 \sigma$ error from the rms noise in a window of $2 N_{\text {win }}+1$ channels centered on that channel. Using this error array, we identified absorption features as series of $n$ channels over which a deviation from the continuum level was observed

individual velocity components in the profiles corresponded to within $\delta_{\mathrm{LOS}}=1.2 \mathrm{~km} \mathrm{~s}^{-1}$. Carilli et al. (2000) argued that $\delta_{\mathrm{LOS}} \sim 10 \mathrm{~km} \mathrm{~s}^{-1}$ is typical of the velocity dispersion of the interstellar medium in galaxies, and that it may even be as high as $\delta_{\mathrm{LOS}} \sim 100 \mathrm{~km} \mathrm{~s}^{-1}$. In order to account for this, we searched for possible absorption lines in a velocity region $\left[-\delta_{\mathrm{LOS}}-\sigma_{\mathrm{DLA}}, \delta_{\mathrm{LOS}}+\sigma_{\mathrm{DLA}}\right]$ centered on the expected DLA frequency with $\delta_{\mathrm{LOS}}=100 \mathrm{~km} \mathrm{~s}^{-1}$ and $\sigma_{\mathrm{DLA}}$ the uncertainty in the DLA redshift. 
Table 4. Candidate molecular absorption systems located within $\approx \pm 200 \mathrm{~km} \mathrm{~s}^{-1}$ of the DLA redshift. $v_{\mathrm{obs}}$ is the observed frequency, $\sigma_{n}$ is the significance of the putative feature, $z_{\mathrm{mm}}$ is the absorption redshift and $z_{21 \mathrm{~cm}}$ is the redshift of the $\mathrm{H}_{\mathrm{I}}$ line (see Curran et al. 2002). The final column gives the offset in $\mathrm{km} \mathrm{s}^{-1}$ between the $z_{\mathrm{mm}}$ and the nominal value of $z_{\mathrm{DLA}}$, with our estimate of uncertainty in the DLA redshift ${ }^{3}$ quoted.

\begin{tabular}{lccrrrr}
\hline \hline DLA & Transition & $v_{\text {obs }}[\mathrm{GHz}]$ & $\sigma_{n}$ & $z_{\mathrm{mm}}$ & $z_{21 \mathrm{~cm}}$ & $z_{\mathrm{mm}}-z_{\text {DLA }}$ \\
\hline B 0248+430 & $\mathrm{CS} 2 \rightarrow 3$ & 105.3701 & 4.2 & 0.39479 & 0.3941 & $190 \pm 20$ \\
B 0458-020 & $\mathrm{HCO}^{+} 2 \rightarrow 3$ & 87.9994 & 3.8 & 2.04045 & 2.03945 & $50 \pm 10$ \\
$\ldots$ & $\ldots$ & 88.0229 & 3.2 & 2.03964 & $\ldots$ & $-30 \pm 10$ \\
$\ldots$ & $\mathrm{CO} 3 \rightarrow 4$ & 151.7144 & 4.3 & 2.03888 & $\ldots$ & $-100 \pm 10$ \\
B 0738+313 & $\mathrm{CO} 0 \rightarrow 1$ & 94.3116 & 4.4 & 0.22224 & 0.2212 & $240 \pm 20$ \\
B 0834-201 & $\mathrm{HCO}^{+} 2 \rightarrow 3$ & 98.5135 & 4.4 & 1.71595 & - & $100 \pm 100$ \\
\hline PKS 1413+135 & $\mathrm{HCO}^{+} 2 \rightarrow 3$ & 214.6110 & 4.6 & 0.24671 & 0.24671 & - \\
\hline
\end{tabular}

Note: the last entry is a known absorption system included as a guide. A spectrum was obtained after $\approx 30$ hours with SEST (August 2001) which may well have been rejected upon visual inspection ${ }^{3}$.

with significance $>\sigma_{\lim }$ standard deviations. Note that the significance of an absorption feature, $\sigma_{n}$, is an overestimate because the flux in adjacent channels is (positively) correlated (each spectral resolution element is sampled at approximately the Nyquist rate, see Murphy et al. (2002) for details).

According to the "expected" $\delta_{\mathrm{LOS}}=100 \mathrm{~km} \mathrm{~s}^{-1}$ velocity differences in the optical and millimetre wave lines-of$\operatorname{sight}^{5}$, the only candidate features identified in our analysis are $\mathrm{HCO}^{+} 2 \rightarrow 3$ and $\mathrm{CO} 3 \rightarrow 4$ towards B 0458-020 as well as the $\mathrm{HCO}^{+} 2 \rightarrow 3$ line towards B 0834-201. As seen in Table 4 , however, increasing the line-of-sight velocity difference to $\delta_{\mathrm{LOS}}=200 \mathrm{~km} \mathrm{~s}^{-1}$ significantly increases the number of candidates. This suggests that either the authenticity of the candidate features is questionable and/or we have underestimated the uncertainties in the DLA redshifts. It is interesting to note that all but one of the candidate lines occur in DLAs with high column densities $\left(N_{\mathrm{HI}} \gtrsim 10^{21} \mathrm{~cm}^{-2}\right)$. The one exception is B 0834-201 which has the highest visual magnitude of these candidates. We emphasise that Table 4 is only intended as a shortlist for follow up observations, which may or may not yield absorption systems at the listed redshifts.

Of all the candidate systems, the most promising and visually striking example of a possible absorption line is the 151.71 GHz feature towards B 0458-020 (Fig. 1), which has a high visual magnitude as well as the highest neutral hydrogen column density of the sample. Comparing this "detection" of $-8 \mathrm{mK}(0.24 \mathrm{Jy}$ at SEST) with the estimated flux density at $152 \mathrm{GHz}(0.62 \mathrm{Jy}$, estimated from the neighbouring values in Table 1) gives an optical depth of $\tau_{\mathrm{mm}} \approx 0.5$. The $\approx 9 \mathrm{~km} \mathrm{~s}^{-1} F W H M$ of the line gives a column density estimate of $N_{\mathrm{CO}} \approx 8 \times 10^{16} \mathrm{~cm}^{-2}$ or $N_{\mathrm{H}_{2}} \sim 10^{21} \mathrm{~cm}^{-2}$, i.e. $\sim 20 \%$ of $N_{\mathrm{HI}}$. This compares well with the column densities and their ratios for the known absorbers, i.e. 10-40\% (Wiklind \& Combes 1994, 1995, 1996c, 1999; Carilli et al. 1998), although it is considerably higher than the rest of the DLA sample.

\section{Summary}

We have observed 18 transitions for molecular absorption over the redshift range $0.2 \gtrsim z \gtrsim 3$ in 11 damped Lyman-alpha absorption systems which lie along the line-of-sight towards radio-loud quasars. Of these, 9 quasars have flux densities

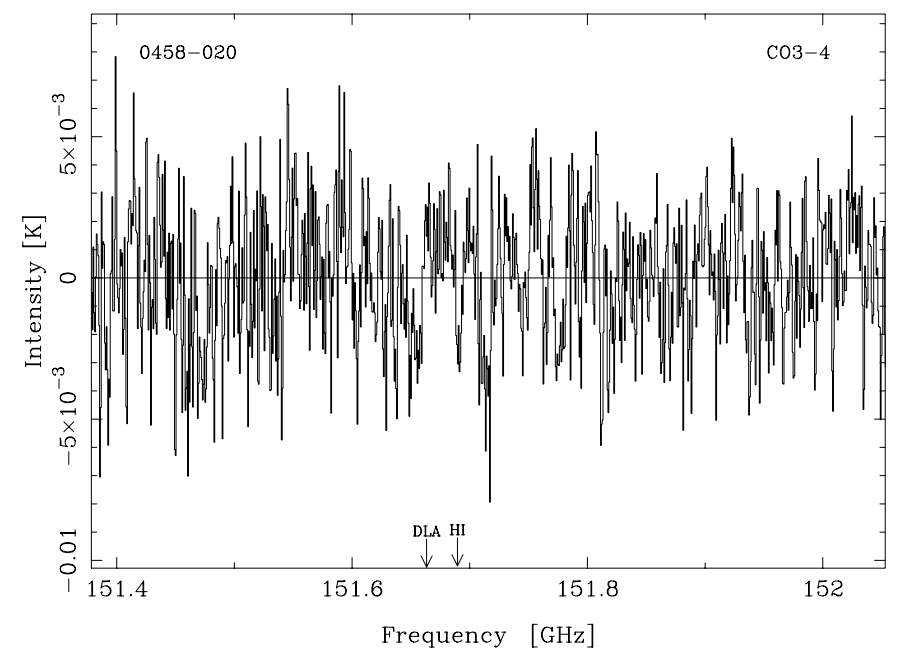

Fig. 1. CO $3 \rightarrow 4$ at $z=2.039$ towards B 0458-020 at the full spectral resolution of $1.38 \mathrm{~km} \mathrm{~s}^{-1}$. The antenna temperature of the peak channel is $8 \mathrm{mK}$ (cf. $\sigma=3 \mathrm{mK}$ ) and the integrated main-beam intensity of the line is $-0.10 \pm 0.02 \mathrm{~K} \mathrm{~km} \mathrm{~s}^{-1}$. The expected frequencies of the line for the (approximate) DLA redshift of 2.0399 and $\mathrm{H}_{\mathrm{I}}$ redshift of 2.03945 (Turnshek et al. 1989; Wolfe et al. 1995) are shown.

$\gtrsim 0.1 \mathrm{Jy}$ in the millimetre band, making our observations sensitive to optical depths $\gtrsim 0.1$ at a $3 \sigma$ level and a spectral resolution of $10 \mathrm{~km} \mathrm{~s}^{-1}$, thus improving the limits for 11 transitions over the previous results.

From these observations and the previously published results, we estimate an upper limit of $\approx 2 \%$ for the molecular hydrogen to neutral atomic hydrogen column density ratio. This is the lower limit derived by Carilli et al. (1996) based on a molecular hydrogen detection and a redshifted $\mathrm{H}_{\mathrm{I}} 21 \mathrm{~cm}$ nondetection. This is consistent with the results of Liszt (2002) who suggests that the low metallicity of the Lyman-alpha system is not favourable for the formation of molecules, but our limit perhaps suggests that we are close to the values necessary to detect absorption lines in at least the high column density $\left(N_{\mathrm{HI}} \gtrsim 4 \times 10^{21} \mathrm{~cm}^{-2}\right)$ DLAs. Subsequently, from an analysis of the spectra we find several significant features located close to the expected redshifts, which do, however, require confirmation. Therefore follow up observations of the candidates given 
in this article should be the next step in searching for the elusive high redshift molecular absorption systems.

Acknowledgements. We wish to thank the John Templeton Foundation for supporting this work. SJC acknowledges receipt of a UNSW NS Global Fellowship and FTR acknowledges support from the Chilean Centro de Astrofísica FONDAP No. 15010003.

\section{References}

Briggs, F. H., \& Wolfe, A. M. 1983, ApJ, 268, 76

Briggs, F. H., Wolfe, A. M., Liszt, H. S., Davis, M. M., \& Turner, K. L. 1989, ApJ, 341, 650

Carilli, C. L., Lane, W., de Bruyn, A. G., Braun, R., \& Miley, G. K. 1996, AJ, 111, 1830

Carilli, C. L., Menten, K. L., Reid, M. J., Rupen, M. P., \& Yun, M. S. 1998, ApJ, 494, 175

Carilli, C. L., et al. 2000, PhRvL, 85, 5511

Combes, F., \& Wiklind, T. 1998, ESO Messenger, 91, 29

Cowie, L. L., \& Songaila, A. 1995, ApJ, 453, 596

Curran, S. J., Webb, J. K., Murphy, M. T., Bandiera, R., Corbelli, E., \& Flambaum, V. V. 2002, PASA, 19, 455

Douglas, N. G., Radford, S. J. E., Roland, J., \& Webb, J. K. 1992, A\&A, 262, 8

Drinkwater, M. J., Webb, J. K., Barrow, J. D., \& Flambaum, V. V. 1998, MNRAS, 295, 457

Foltz, C. B., Chaffee, F. H., \& Black, J. H. 1988, ApJ, 324, 267

Ge, J., \& Bechtold, J. 1997, ApJ, 477, L73

Ivanchik, A. V., Potekhin, A. Y., \& Varshalovich, D. A. 1999, A\&A, 343, 439

Le Brun, V., Bergeron, J., Boisseé, P., \& Deharveng, J. M. 1997, A\&A, 321,733

Ledoux, C., Srianand, R., \& Petitjean, P. 2002, A\&A, 392, 781

Levshakov, S. A., Molaro, P., Centurión, M., et al. 2000, A\&A, 361, 803

Levshakov, S. A., Dessauges-Zavadsky, M., D’Odorico, S., \& Molaro, P. 2002, ApJ, 565, 696
Liszt, H. 2002, A\&A, 389, 393

Lovas, F. J. 1992, J. Phys. Chem. Ref. Data, 21(2), 181

Murphy, M. T., Webb, J. K., Flambaum, V. V., et al. 2001a, MNRAS, 327,1244

Murphy, M. T., Webb, J. K., Flambaum, V. V., et al. 2001b, MNRAS, 327, 1208

Murphy, M. T., Webb, J. K., Flambaum, V. V. Churchill, C. W., \& Prochaska, J. X. 2001c, MNRAS, 327, 1223

Murphy, M. T., Curran, S. J., \& Webb, J. K. 2002, MNRAS, submitted

Petitjean, P., Srianand, R., \& Ledoux, C. 2002, MNRAS, 332, 383

Srianand, R., Petitjean, P., \& Ledoux, C. 2000, Nature, 408, 931

Turnshek, D. A., Wolfe, A. M., Lanzetta, K. M., et al. 1989, ApJ, 344, 567

Webb, J. K., Flambaum, V. V., Churchill, C. W., Drinkwater, M. J., \& Barrow, J. D. 1999, PhRvL, 82, 884

Webb, J. K., Murphy, M. T., Flambaum, V. V., et al. 2001, PhRvL, 87, 091301

Wiklind, T., \& Combes, F. 1994a, A\&A, 286, L9

Wiklind, T., \& Combes, F. 1994b, A\&A, 288, L41

Wiklind, T., \& Combes, F. 1995, A\&A, 299, 382

Wiklind, T., \& Combes, F. 1996a, in ESO Symposia (Springer), ed. P. Shaver, 86

Wiklind, T., \& Combes, F. 1996b, A\&A, 315, 86

Wiklind, T., \& Combes, F. 1996c, Nature, 379, 139

Wiklind, T., \& Combes, F. 1997, A\&A, 328, 48

Wiklind, T., \& Combes, F. 1998, ApJ, 500, 129

Wiklind, T., \& Combes, F. 1999, in Highly Redshifted Radio Lines, ASP Conf. Ser., 156, 202

Wiklind, T., \& Combes, F. 2001, in Gravitational Lensing, ed. T. G. Brainerd, \& C. S. Kochanek (San Francisco: Astronomical Society of the Pacific), ASP Conf. Proc., 237, 155

Wolfe, A. M., Briggs, F. H., Turnshek, D. A., et al. 1985, ApJ, 294, L67

Wolfe, A. M., Lanzetta, K. M., Foltz, C. B., \& Chaffee, F. H. 1995, ApJ, 454, 698 